\title{
Intelligent Adaptive Presentation and E-testing System based on User Modeling and Course Sequencing in Virtual Classroom
}

\author{
Nidal A.M Jabari \\ ITS - Surabaya \\ Indonesia
}

\author{
Mochammad Hariadi \\ ITS - Surabaya \\ Indonesia
}

\author{
Mauridhi Hery Purnomo \\ ITS - Surabaya \\ Indonesia
}

\begin{abstract}
This paper introduces a solution for the problem of dealing with the student personalization in e-learning and virtual classrooms. Dual problem relating to LOs presentation in the best appropriate way to the student and conduct the tests in a way that allows him to answer according to his cognitive style and giving him more trials to answer. IMS SS describes the organization of LOs in hierarchical manner, as a tree allows to deliver LOs in sequence and more verifiable. Petri Net offers the ability of using conditions within the learning process and then respond to the result of the condition. learning environment is divided into three layers. DM, AM and RM. Through the relationship and interaction between these three layers we can control the sequence and organization of the learning process . Student model is the core of adaptive learning since it determines the behavior of the system adaptive process . It has been studied the most important student characteristics required in the adaptive process mainly the different patterns of cognitive styles . This paper discussed the design of the adaptive learning process basing on these concepts. We got integrated design provides learning activities either LOs or tests in sequence and adaptive to the student cognitive style. This system evaluates the knowledge of the student beside his new cognitive style which should be used in the next concept presentation. The paper discusses also the full environment of this system through the design of models required to achieve the objective as well as services, infrastructure, and some additional services that can get benefit from the system such as adaptive chat room .
\end{abstract}

\section{Keywords}

adaptivity, cognitive style, domain, exercise, stereotype

\section{INTRODUCTION}

In recent learning systems, technology strongly involved in the pedagogical process, especially in the virtual classroom, virtual classroom contains mainly two components, first is the lectures presentations, second the student evaluation techniques .Most learning systems manage these two processes in traditional way .Both lectures presentation and evaluation process are introduced in the same way for all students .Personal differences among students specially student preferable way of study are not considered in these systems .

Data about student is needed to build the student model and estimate the student knowledge level and learning style [1].This evaluation will be reflected to the adaptivity of the learning objects presentation. In this paper we introduced technical solutions for adaptive learning based on two sources of student data. The questionnaire answered by the student at the beginning of the course, which will give an initial stereotype for the student, needing to have initial data about the student is an important factor to tell the system how to deal initially with each student in private manner .Later the system should be able to update the student stereotype dynamically during the learning process . The exam follows each concept presentation is used to evaluate the student knowledge and the new cognitive style .

An integrated model for adaptive e-learning system is an important factor . It's important to keep models in the system separated from the system processing. Data storage, domain and user models are kept separately from the system to allow other systems which need to use SM and DM to use them independently

The rest of the paper is the following, section for 2 discusses related work, section 3 discusses our model, section 4 discusses the conclusion and future work, section 5 for acknowledgement .

\section{RELATED WORK AND BACKGROUND}

\subsection{Adaptive course sequencing}

Learning activities in e-learning are in their nature multiple, simultaneous and sequential[2] . Some are learning objects (LOs) while others are exams, quizzes or other learning medias .Through this concept we can get different paths within the learning process for each concept. This sequence has been studied by Bailey so-called IMS SS as seen in figure 1.

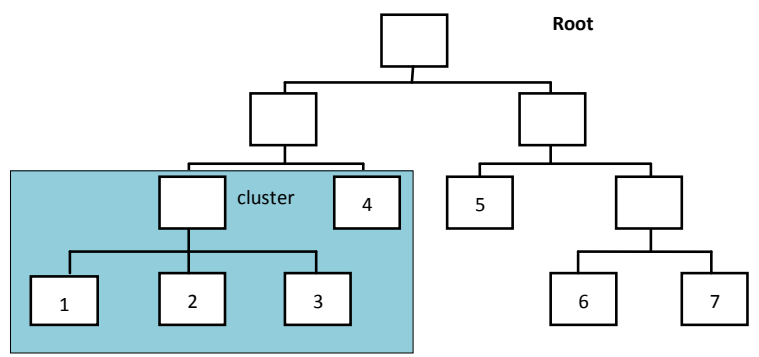

Fig 1 : sequencing activity tree

Getting different paths in one group or to get different patterns of formative assessment is suitable for applications in different areas of e-learning as we have different student with different characteristics. IMS SS can decide the next step possible to be either LO , examination ,quiz or other learning media as seen the tree cluster in figure 1.The main objective of sequencing is to get the best appropriate learning strategy to the learner. Because the learning activities constitute a hierarchy in its composition, we are able to form different models of learning 
activities. This

can

be

done through. 1) repeated random selection of LO until the student gives evidence of knowledge mastery . 2) selection of the next LO . 3) Basing of the student profile a group of LOs can be selected . 4) As goal oriented systems, the goal will determine the set of LOs which meet the current goal and discover the learner skill [3]. A parent activity and its children are referred to as a cluster of activities .

\subsection{Course sequencing behavior model presented by Petri net}

Petri Net is a directed graph consists of the nodes represent transitions such as an event. And places such as the conditions expressed in the circles. Directed arcs reflect the event before or after the condition. Petri Net has modeling tools such as the other modeling standards , provides graphical notations like choice, process, iteration , and concurrent execution. However, Petri Net has a mathematical definition for the implementation in addition to well proved theories [4] . Madjarov and Betari studied this model through cluster of sequential behavior. They found that the breakdown of learning material have to deal with the various activities which are delivered to the learner as managed sequences .

Madjarov and Betari introduced a model based on Petri Net in the form of a hierarchical structure of learning activities (LOs) [6] . Learning material is organized as a cluster tree and the sequence in it depends at the disposal of the learner or the response to exercises designed to determine the level of the student, which can be determined through the examination at that stage or level. Service provided to students through LOs are more suitable to the level of knowledge of the student. This form is more dynamism in the sequential behavior and sequencing rules of schema , which ensures more integration of various types of LOs in the sequencing rules of schema [6] . The course cluster sequencing is illustrated in figure 2 . Concept in the domain, there must be a range of activities

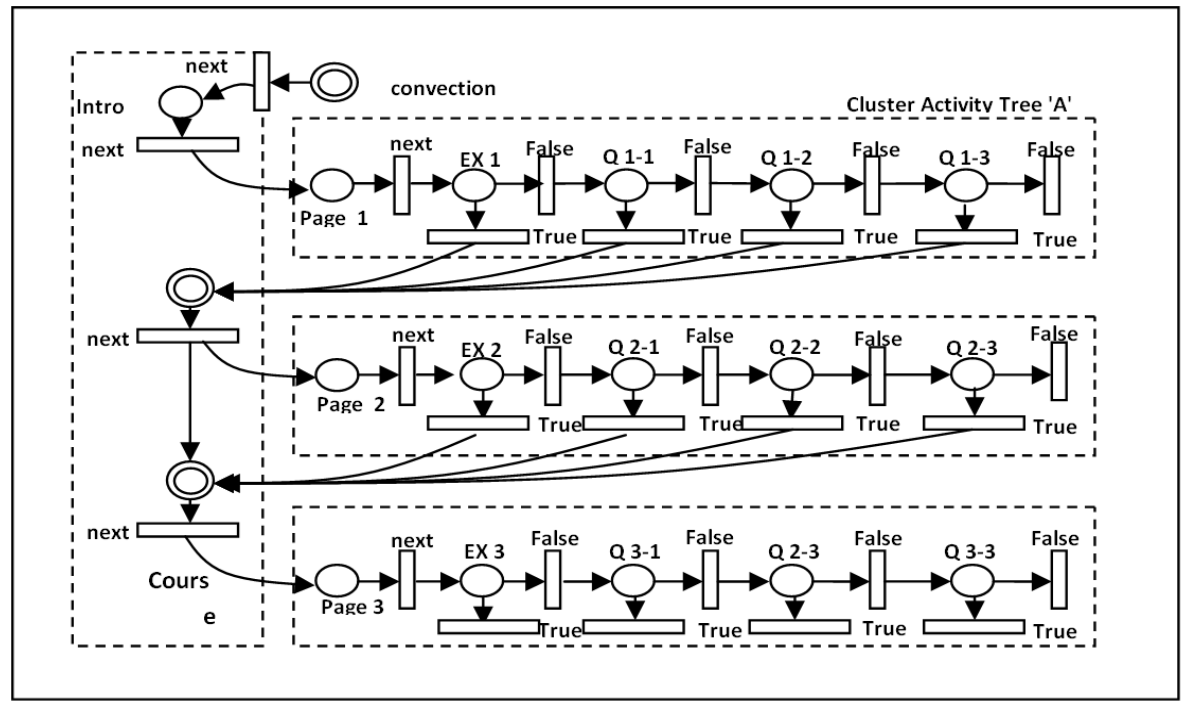

Fig 2 : course cluster sequencing behavior presented by A Petri Net

In our model, we can get the benefit from this concept, as we face many of the conditions and the transition from situation to situation within the learning process, so this model will be a main pillar in our model

\subsection{Learning model based on Pedagogy}

The aim of learning is to get knowledge. To acquire knowledge, the learning activities such as learning content or evaluation activity must be supported by some of the resources. Zhang in [5] divided the learning process into three layers. Each layer to achieve a certain objective. The first layer related to knowledge structure for the students called the Domain model (DM). In most cases, the domain is divided into several parts from the top to the lowest. For example, can be the division of the unit into the subunit into the concept. The concept is the smallest learning unit may be atomic or composite of several concepts. Domain model is not the storage structure of the learning materials, but the term only about the structure of the LOs. The second level related to the activity structure called activity model (AM ). Each supporting it.

These activities may be a review of prior knowledge, learning content or assessment. The third layer of concepts related to the resource description called resource model (RM). This layer represents the learning materials of the course. Adaptation strategy based on these three layers. But cannot be adaptive if we don't focus on information related to the student which is called student model as seen in figure 3 .

LOs are made up of concepts as previously mentioned . These concepts must have a clear relationship between them. For each concept there are a range of activities related to this activity and strategy for the selection of these activities.

As we will see in our proposed model we also build our conceptual model on these three layers. The interaction between these three layers will be describe clearly .

\subsection{Cognitive styles}

As mentioned earlier, the student model is the main pillar in the adaptive learning process . Because the system must be acting according to the characteristics of a student known already to the system. This Model generally has inferred information $[7,8]$. One of the most important characteristic 


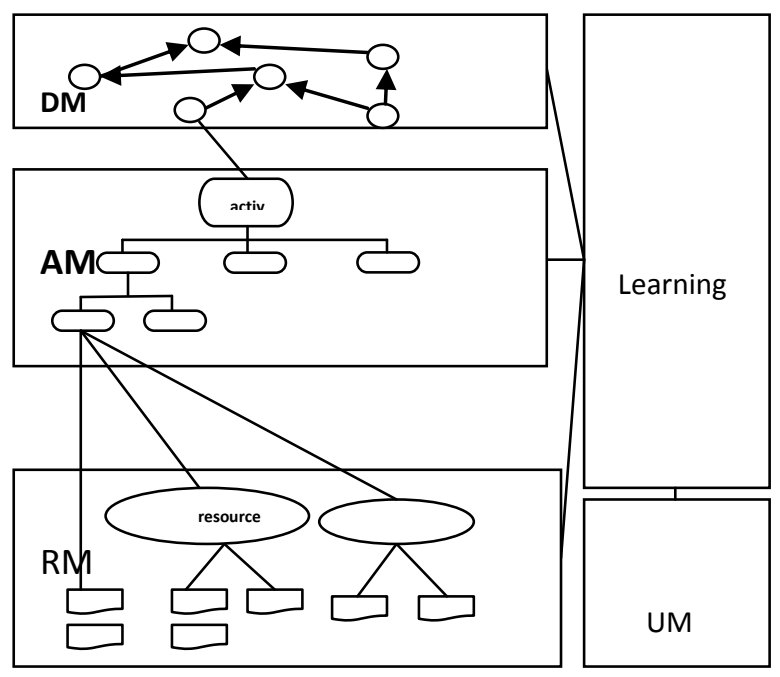

Fig 3 :the learning model based on pedagogy

that should be taken into account is the student cognitive style. Many researchers discussed in this area to get the main cognitive styles of the student. In [9] defined cognitive style as the way of the student learning by which the learning materials is displayed so that the student should be in the best degree of comprehension and understanding. The system should provide learning materials basing on the cognitive style for the student to ensure maximum knowledge to him. As stated in [10], the most important cognitive styles are: - Verbalisers "consider the information they read, see or listen to, in words or verbal associations"(10) .

- Imagers on the other hand, when they read, listen to or consider information, experience

"fluent spontaneous and frequent pictorial mental pictures" (10) .

- Analytics according to Riding will take a structured approach to learning and will prefer in $r$ information that is set out in a clearly organized way .
- Wholists on the other hand will not habitually take

a structured approach and may therefore need help in imposing a structure

on some unstructured situations or experiences formation that is set out in a clearly organized way.

\section{INTELLIGENT ADAPTIVE PRESENTATION AND E_TESTING SYSTEM BASED ON STUDENT MODELING AND COURSE SEQUENCING}

Our proposed system will be also web based oriented, web server with database system .To take advantage of previous studies that have been discussed earlier in this paper .The proposed system will work in integrated environment of the last discussed studies .Selecting path of learning objects according to the student cognitive style which is saved and updated dynamically in the student profile .In our model we consider only 3 types of cognitive style .It is

possible to use more types when required . They are verbal (ver.), visual (vis) and analyst (ana) .They are represented in the set $<$ ver, vis , ana $>$. Sequences of LOs and other activities are organized in a tree form .Visiting the course content starting from chapter until the smallest entity the concept .The student will be given a several chances to answer the same exercise. The exercise will be introduced in several patterns to let the student have more chances to choose the right answer .First trial always should be in the current student cognitive style while the others will be in different styles in order to estimate the new student cognitive style dynamically . Using (condition / event) state in Petri Net to decide the next step after the answer of the student . Basing on exam results, the student will be directed to the next concept or keep trying to understand the current concept . Determining the exact student knowledge level about the current concept is obtained through completion of the exam and get a score. This will serve for two goals , first the evaluation of the student knowledge academically, second to provide information to the student profile, this information beside other information as seen in [11] will serve for adaptive learning and other systems need information about the student like adaptive discussion room .

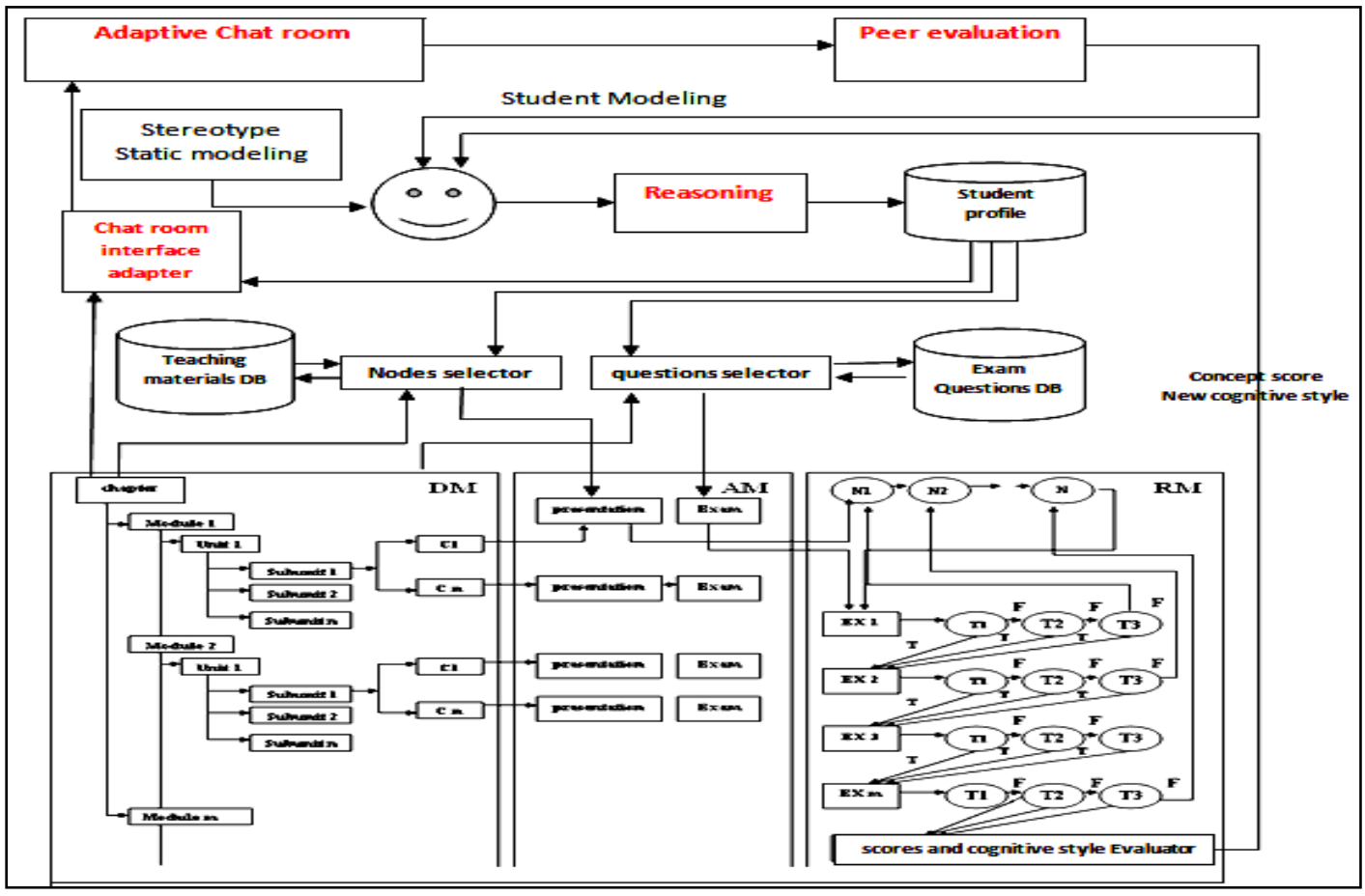

Fig 4 : the proposed model 
The overall view of the system is shown in figure 4 .The system consists of three layers, Domain model , Activity Model and resources model .A brief description for each model in the following points :

\section{DISCUSSION}

\subsection{Domain Model}

Domain model in as described in [11] is a set of chapters $(\mathrm{CH})$, each chapter consists of a set of Modules (M),module is separated into several unites (U) and unit to several subunits (SU), the subunit has several concepts which represent the learning objects of this subunit, the student should follow these concepts one by one in sequence to achieve the require knowledge required for this subunit .Each concept should be accompanied with expert estimation of the required knowledge to pass this concept in addition to the cognitive style related to this concept. In such a way we can define the concept as the set < name, path , expert level , cgs (ver, vis ,ana) $>$. For example we can define abacus concept in 101 course as the tuble < abacus, intro-counting-machines, $77 \%$, ver $>$. Expert knowledge serve to decide if the student passes the concept or should be keep studying the same concept. Cognitive style serves to decide how to present the concept LOs to the student. The Domain model doesn't have any teaching materials .It is only describes the structure and the relationships between course components. Domain model will provide information to several modules in the system .Nodes selector, Question selector, Chat room interface adapter. These modules will be discussed later in this section .

\subsection{Student Model}

Student Model is the core of the learning adaptive systems and ITS . It will provide the required data for adaptation techniques beside the domain model for both presentation and exam agent .Student model actually consists of several sources of data in our system we concentrate in two sources : -Static student modeling (Stereotype):

A stereotype is a popular belief about specific types of individuals. Stereotypes are standardized and simplified conceptions of groups based on some prior assumptions. In our system there are several characteristics and preferences can be considered in the system which affect the behavior of the system. These preferences are called domain independent .This data mostly static and not changeable during the instructional process .For example and not all we can consider preferable time of learning within the day or night, sex , age ,background, skills, etc .This data can be gathered directly from the student via a questionnaire, and used to classify the students into groups called stereotypes. Then store this data in the student profile database. In this paper we will consider only one entry in the stereotype, the student cognitive style as the student use the system for the first time .

-Dynamic Student modeling (Overlay Model):

Once the student starts to use the system his data will be gathered using dynamic modeling. Dynamic modeling has two entries ,one related to student the knowledge estimation through exam evaluation and peer evaluation .The other is related to the student new cognitive style. These both modeling processes are described in the following sections .

-Activity Model (AM) :

AM will be the second process right after the student login to the system .For each concept there are two or more activities .In our model we used only two activities, first is the Presentation Agent (PA), second is Exam Agent(EA) . AM will have mainly the following functions:

-Determine Last studied concept score from SM .
-Determine Expert minimum score accepted from Domain model.

- Compare the student score with the expert minimum accepted score and determine if the student can move to a new concept or repeat the same concept (overlay model) .

-Determine the new concept to be taught from domain model (current or new ).

- State the student current cognitive style from SM .

Generally AM will organize the relationship between DM and RM , in such way we can control the sequencing of the learning objects in addition to their required exams in correspondent with the sequence of the DM concepts sequence .

PA will have the function of generating the query to retrieve the appropriate nodes to built the LOs presentation for the current concept and passing this query to the Nodes selector to retrieve the required nodes .Then gives an order to the resource model to adjust the selected nodes and present them as pages to the student .PA input and output described in figure 5 .

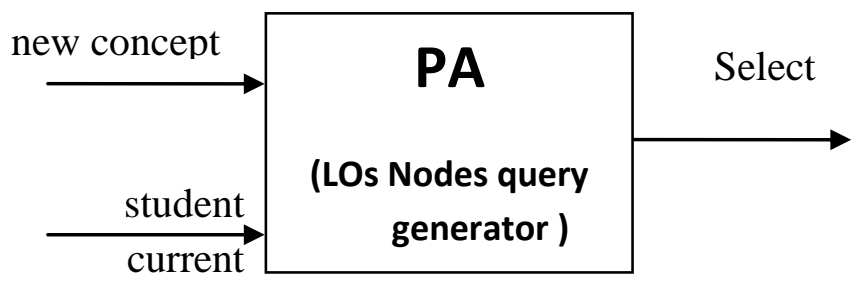

Fig 5 : Presentation agent modeling

EA, in its turn receives as input the student current cognitivestyle , and current concept to be tested which is already determined in AM .EA also will generate the query to retrieve the appropriate questions to evaluate the student for current concept .Pass the query to the Questions selector to retrieve the required questions from Questions Database (QDB) which match with these parameters . EA will give an order to the RM to present the questions in sequence to the student .EA has similar model of PA modeled in figure 5 .

The question which will be presented to the student has the form as in the set <concept, Question, Trial, Question Text ,cognitive style $>$ as seen in figure 7 .For each question Trial there are three answers represented in the tuple <concept, Question, Trial, Answer i> as seen in Trial tree in figure 8 .

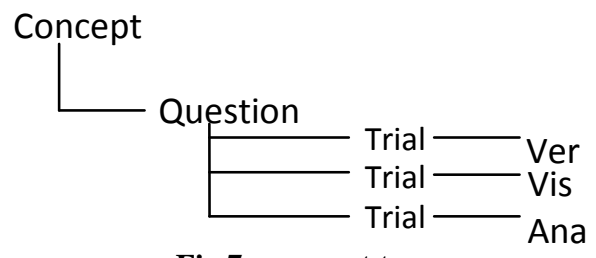

Fig 7 : concept tree

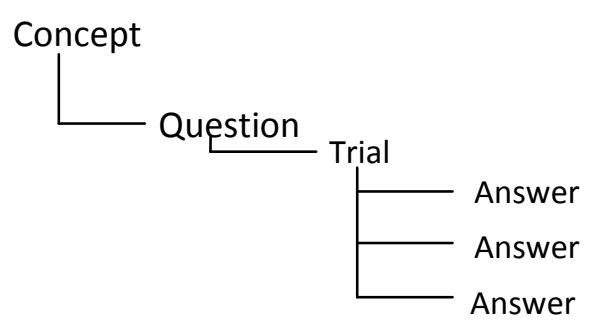

Fig 8: Trial Tree 
These questions will be presented to the student after finishing the concept presentation nodes. Exam agent also will match between the student answer with the right answer and determine the score according to the trial .If the student answer the question correctly then he will get the score of (10/trial no) .If the student fails to answer in three trials EA will send him back to the presentation agent which will enforce the student to review the Nodes related to this concept again. Then back to the test questions again.Another function of Exam agent is determine the new cognitive style of the student .This determination will be extracted from the field of cognitive style assigned to each question trial .Exam agent will count the number of trials which has one cognitive style most answered correctly and state the new student cognitive style .

RM consists of forms which should be built dynamically according to the order of the PA and EA. These forms will be as the result of the queries matched with the parameters passed by PA and EA to QS and NS. AM activities are described in flow chart shown in figure 6 .

- Nodes Selector :

Nodes selector is an a query executer which receive the query from PA and execute it .The query will be in the form of :

Select Nodes from Materials_DB where

Concept $=$ current_concept in AM AND

Cognitive_style $=$ current_CGS in AM

$\mathrm{RM}$ will receive these nodes and present then in sequence

- Question Selector :

Question selector is also receive a query from EA and execute it

The exam query in the style like :

Select EX Trial_text, Trial_right_answer,Trial_choices

from Exam_Question_DB where

Concept $=$ current concept in $A M A N D$

Cognitive_style $=$ current_CGS in AM AND

Trial_no=Current_trial_no

RM will present the questions and the answers to the student in sequence .

- Concept Score Evaluator :

As seen in the system flow chart, Concept evaluator is incremental procedure read the student answer and compare it with the right answer .Concept evaluator should take in consideration the trial of the question to calculate the concept score .The concept evaluator is described as the set < concept ,question, Trial, right answer, student answer, result, score $(10 /$ Trial \# $)>$ as seen in figure 9.Concept evaluator will evaluate all exercises related to the current concept .Concept evaluator will represent one part of the dynamic modeling of the student since the concept score will be stored in the student profile once it is processed .

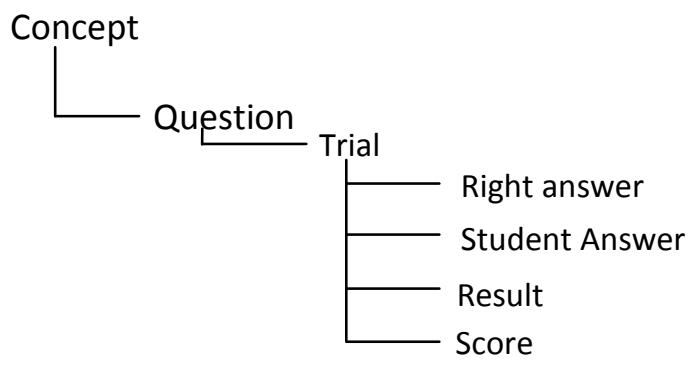

Fig 9 : New Trial Tree
- Cognitive style evaluator :

As seen in the system algorithm, Cognitive style evaluator is another accumulative dynamic student modeling. It evaluate the new student cognitive style according to his questions answers. The cognitive style evaluator will have the following procedure .

For each exercise trial in the exam related to once concept there is one answer .This answer has assigned cognitive style .When the student answer the cognitive style of this question is stored .At completing of all exercises each cognitive style will has a rate percent . For example if the exam has 15 questions. The student answered 8 of them with 'ver' cognitive style , 5 with 'vis' cognitive style , and 2 with 'ana' cognitive style. It means the student has the set $<53 \%, 33 \%, 14 \%>$ for the set <ver, vis, ana .At this stage we can consider the student as verbal student in this concept .In the next chapter presentation agent will generate a query which look for verbal LOs. However in the next concept the student will repeat the same process and will get a new set of cognitive style let's say $\langle 34 \%, 55 \%, 11 \%\rangle$. In this case next concept will be presented in visual pattern. At the end of the unit the student cognitive style for this unit is calculated incrementally from all previous cognitive styles measured in last unit concepts. This process will be repeated incrementally until finishing the chapter. Fuzzy logic which will be describes in next research paper will be used to estimate the student cognitive style for the next chapter. For more details about this algorithm we can describe it in the following flow chart in figure 10 .

- Chat room Interface adapter :

Chat room interface adapter is discussed in our last paper [Jabari] .It is an additional separated system can get advantage from student modeling in our system. Adaptive chat room is a collaboration tool among students when they want to discuss among each other's .This tool gives an evidence about the student knowledge level for each student participate in chat room relying on student-concept evaluation. In order to give this evidence the chat room take an input from both student and domain model, and adapt the chat room according to the input. The student who has knowledge less than the current student will appear in red color. The student who has knowledge equals to the current student knowledge will be given a yellow color, and finally the student who has higher knowledge will have green color .This way make it easier for the current student to determine with which student he should discuss the current concept

- Peer evaluation :

Actually the student will be evaluated for each concept separately through the exam after each concept learning process . For example student X got a score of $60 \%$ in concept $\mathrm{C} 1$ evaluation. During discussion with peers he may have new knowledge about the concept .This increment in knowledge level should be measured. Once two peers finishing their discussions about one concept, a questionnaire should be popped up for both peers to evaluate each others , after filling the questionnaire it will be analyzed and passed to the student model to extract the increment in the students' knowledge and store it in the student profile .

- Algorithm evaluation

To evaluate the algorithm let's consider the following example We have the course introduction to computer science

First : DM (partially)

Chapter 1 : introduction to computer

Module 1 : introduction 


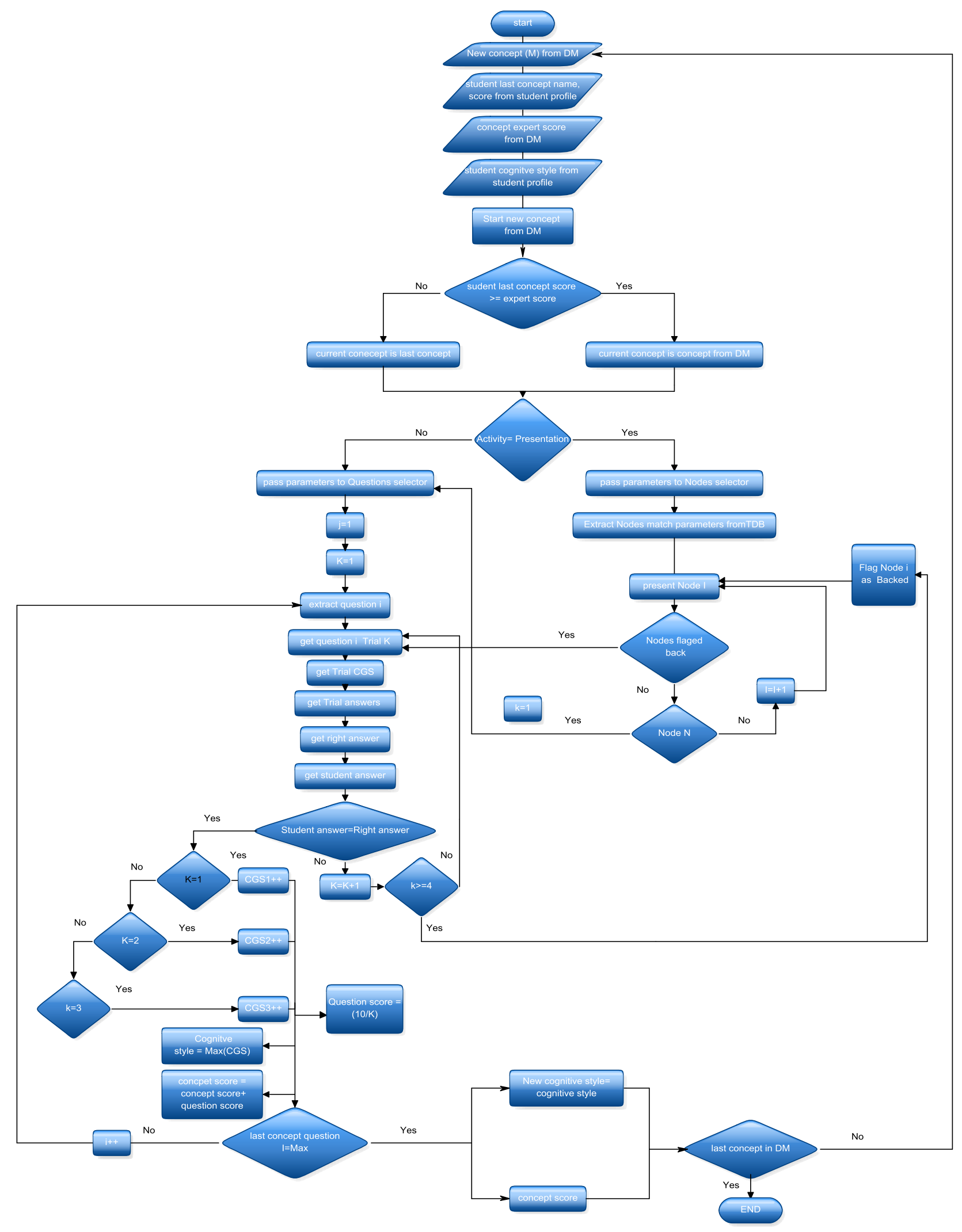

Fig 6 : AM activities 


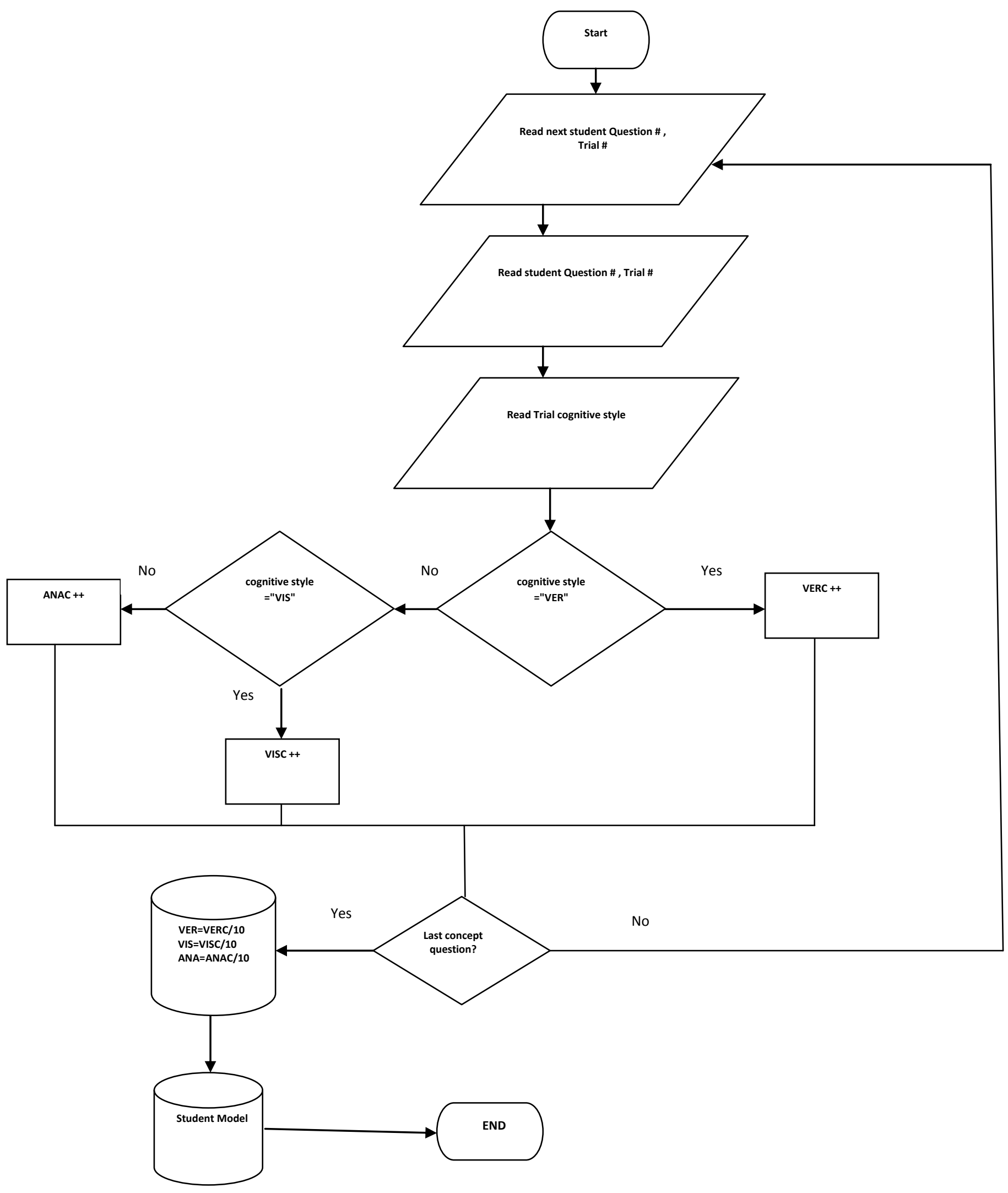

Fig 10 : Cognitive style evaluator for one concept

Module 2: computer ethics

Module 3: comp2uter development

Unit 1 :manual counting

Subunit $1:$ devices

Concept $1:$ abacus
Concept 2 : Napier

Concept $3:$ outghtred Subunit 2 : comparison

Unit 2 :half mechanic counting Unit 3 :electronic computers 
Chapter $2:$ computer system components

Chapter 3 :software

Chapter 4 : internet

For concepts from 1 to 3 in subunit 1 , unit 1 , module 3 , chapter 1

let's consider the student initially has cognitive style VER .

This estimation can be extracted from a questionnaire .Nodes (web pages ) related to each concept is already stored in the teaching materials database in different forms for different cognitive style and related directly to DM architecture, let's take concept 1 : Abacus as an example, two nodes as a sample related to this concept will be as the following figure 8 .

For each Node there are three patterns . N1 (N11 ,N12 ,N13) and (N21, N22, N23) for <VER, VIS , ANA > respectively. Since the student initially has cognitive style as 'VER' Then the PA will generate the query to select Verbal Nodes . Nodes selector will retrieve $\mathrm{N} 11$ and $\mathrm{N} 21$ for this concept .RM will present these two concepts in sequence to the student. As a sample we consider here only these two nodes. Once the student finished the nodes he should directly start the exam related to this concept .Test related to each concept is already stored in the Test database. EA will receive current concept from AM .In our example its abacus, and the student cognitive style is 'VER' and generate the query . Questions selector will execute the query and get the questions and trials .In figure 9 we have the result of the query retrieved by

\begin{tabular}{|c|c|c|c|}
\hline & N11 & N12 & N13 \\
\hline N 1 & \begin{tabular}{l} 
Definition \\
\multicolumn{1}{c}{ Abacus } \\
was \\
invented in \\
china, it's \\
just a \\
simple \\
machine \\
consists of \\
a columns \\
in vertical \\
positions \\
full of \\
small rings
\end{tabular} & $\begin{array}{l}\text { Definition } \\
\text { Abacus was } \\
\text { invented in } \\
\text { china, its look } \\
\text { like as in the } \\
\text { picture }\end{array}$ & $\begin{array}{l}\text { Definition } \\
\text { Abacus } \\
\text { was invented } \\
\text { in china, } \\
\text { See the video } \\
\text { of this device } \\
\text { and it works } \\
\text { on the } \\
\text { following } \\
\text { web site } \\
\text { www.countin } \\
\text { gmachin.com }\end{array}$ \\
\hline
\end{tabular}

\begin{tabular}{|c|c|c|c|}
\hline & $\mathrm{N} 21$ & $\mathrm{~N} 22$ & $\mathrm{~N} 23$ \\
\hline $\mathrm{N} 2$ & $\begin{array}{l}\text { How does it } \\
\text { work } \\
\text { Using rings } \\
\text { to represent } \\
\text { place } \\
\text { values }\end{array}$ & 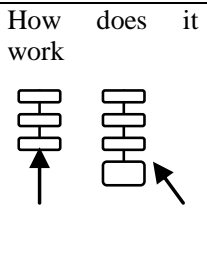 & $\begin{array}{l}\text { How does it } \\
\text { work } \\
\text { Flash } \\
\text { representation } \\
\text { for how this } \\
\text { machine } \\
\text { perform the } \\
\text { calculation }\end{array}$ \\
\hline
\end{tabular}

Fig 11 : Multi paths of learning objects

\begin{tabular}{|c|c|c|c|}
\hline & T1 & $\mathrm{T} 2$ & T3 \\
\hline Q1(N1) & $\begin{array}{l}\text { What } \\
\text { abacus }\end{array}$ & $\begin{array}{l}\text { Select what are } \\
\text { you seeing in } \\
\text { the shown } \\
\text { picture }\end{array}$ & $\begin{array}{l}\text { Select how you } \\
\text { can create } \\
\text { abacus machine }\end{array}$ \\
\hline CGS & VER & VIS & ANA \\
\hline Choices & $\begin{array}{ll}\text { A. } & \text { some } \\
\text { answer } & \\
\text { B. } & \text { some } \\
\text { answer } & \\
\text { C. } & \text { some } \\
\text { answer } & \end{array}$ & $\begin{array}{l}\text { A. some answer } \\
\text { B. some answer } \\
\text { C. some answer }\end{array}$ & $\begin{array}{l}\text { A. some answer } \\
\text { B. some answer } \\
\text { C. some answer }\end{array}$ \\
\hline $\begin{array}{l}\text { Right } \\
\text { Answer }\end{array}$ & $\mathrm{A}$ & B & B \\
\hline Q2(N2) & $\begin{array}{l}\text { Look at the } \\
\text { rings in the } \\
\text { picture and } \\
\text { select the } \\
\text { number } \\
\text { represented }\end{array}$ & $\begin{array}{l}\text { In the following } \\
\text { video select the } \\
\text { number counted } \\
\text { by John }\end{array}$ & $\begin{array}{l}\text { How does } \\
\text { abacus work }\end{array}$ \\
\hline CGS & ANA & VIS & VER \\
\hline Choices & $\begin{array}{lr}\text { A. } & \text { some } \\
\text { answer } & \\
\text { B. } & \text { some } \\
\text { answer } & \\
\text { C. } & \text { some } \\
\text { answer } & \\
\end{array}$ & $\begin{array}{l}\text { A. some answer } \\
\text { B. some answer } \\
\text { C. some answer }\end{array}$ & $\begin{array}{l}\text { A. some answer } \\
\text { B. some answer } \\
\text { C. some answer }\end{array}$ \\
\hline $\begin{array}{l}\text { Right } \\
\text { Answer }\end{array}$ & $\mathrm{B}$ & $\mathrm{A}$ & $\mathrm{C}$ \\
\hline Q3(N3) & Some Q & Some Q & Some Q \\
\hline CGS & VIS & ANA & VER \\
\hline & $\begin{array}{ll}\text { A. } & \text { some } \\
\text { answer } & \\
\text { B. } & \text { some } \\
\text { answer } & \\
\text { C. } & \text { some } \\
\text { answer } & \\
\end{array}$ & $\begin{array}{l}\text { A. some answer } \\
\text { B. some answer } \\
\text { C. some answer }\end{array}$ & $\begin{array}{l}\text { A. some answer } \\
\text { B. some answer } \\
\text { C. some answer }\end{array}$ \\
\hline $\begin{array}{l}\text { Right } \\
\text { Answer }\end{array}$ & $\mathrm{C}$ & $\mathrm{C}$ & A \\
\hline
\end{tabular}

\section{Figure 12 : Multi paths of learning objects and multi trials} of exam questions

Questions selector

EA will pass the questions trials to the RM in sequence Question 1 first trial according to his answer he either go to next question or receive next trial . Suppose the student answered question 1 trial 2 correctly then he will get the score of $(10 / 2=0.5)$.Next question will be presented to the student . Second question the student answers Trial 2 .The score is also 0.5 .In the last question the student answers Trial 3 .The score is $(10 / 3=3.33)$. Final score of this concept is $(0.5+0.5$ $+0.33=1.33)$. The student answered two question with 'VIS' ( degree of Visual is $2 / 3=0.67$ ) and one question with 'VER' (degree of Verbal is $1 / 3=0.33$ ) .The student dynamic modeling gives two sets for this concept . concept score < $1.33>$, concept cognitive style $<0.33,0.66,0>$ for $<$ VER , VIS , ANA > .The new student cognitive style will be VIS and stored in student profile. AM will receive this cognitive style from the student profile and pass it to PA to use it for the next concept . 


\section{CONCLUSION}

A prototype for student adaptive online presentation and evaluation based on learning objects sequencing and Petri Net model is a strong tool for adaptive e-learning approach, the algorithm which is introduced contains the evaluation tool for student knowledge and estimation the student cognitive style, the model presents the learning objects according to this style ,cognitive style will be initially determined by the questionnaire proposed by [7], then this cognitive style will be updated dynamically by the exam questions sections, these sections will be extracted from the questions types, each section will give indication about one type of cognitive style, exam should be applied according to the concept learning objects, each question can be presented in different forms to give the student many chances for better understanding the question and try to answer it several times, each time he fails he will lose score, if he fails to answer the evaluation from one level to level until having the evaluation for all the course.

\section{FUTURE WORK}

In the future work, the implementation model for the learning objects in RM will be described in detail, the mechanism of AM and how it should selects the learning objects according to cognitive style.

\section{ACKNOWLEDGEMENT}

The Researcher thanks the Indonesian government for the support of his research, and the Ministry of Learning in Indonesia for covering the study through the scholarship.

\section{REFERENCES}

[1] Sasakura , Yamasaki, "A framework for adaptive elearning systems in higher learning with information visualization ", IEEE ,2007.
[2] Warwick bailey, " IMS simple sequencing " , JISC , 2008

[3] Blackmon W ., Rehak D . "customized Learning :A web services approach ,proceeding of EDMEDIA, 2003.

[4] Rachid Hamadi Boualem Benatallah , "Petri Net-based Model for Web Service Composition " ,School of Computer Science and Engineering, The University of New South Wales ,2003.

[5] Juinghau Zhang , " Dynamic Generation of the Adaptive Courseware Based on Learning Technology Standards" , the fifth international conference on computer science and learning ,2010, p 741-745.

[6] Madjarov , Betari , " Adaptive learning sequencing for course customization :A web service Approach ", IEEE 2008 , p $530-535$.

[7] Liu , Tang , Ma ," A rule-based Approach for student modeling ", IEEE , 2008 , p 526-530.

[8] Zhiping , Sun $\mathrm{Yu}$, "Agenet-based knowledge management in intelligent tutoring system ", the fifth international conference on computer science and learning , 2010 , p $578-582$.

[9] J. L. Edwards , "Cognitive Style Assessment and Adaptation to User Style in the

LOCATE Workspace Layout Design Tool " , : Artificial Intelligence Management and Development Corporation (AIMDC), AC279 , 2006 .

[10] Riding, R.J. "Personal Style Awareness and Personal Development". Birmingham:Learning and Training Technology,(1994).

[11] Jabari , Hariadi , " adaptive web - based virtual classroom based on student modeling " , ITS , 2010 . 